\title{
A BIAS IN ESTIMATION OF VIABILITIES FROM COMPETITION EXPERIMENTS
}

\author{
D. ANXOLABEHERE*, J. M. GOUX and G. PERIQUET* \\ Laboratoire de Génétique des Populations, Université Paris VII, 2 place Jussieu, \\ 75005 PARIS, France
}

Received 12.vii. 81

\section{SUMMARY}

\begin{abstract}
Some current estimators of the relative viabilities of genotypes used in competition experiments, are shown to be biased, the bias being a function of the sample size and the gene frequency. From their use, an erroneous conclusion of a frequency-dependent phenomenon can be drawn. Almost unbiased estimators are advocated. The calculation of the exact distribution of these estimators for each experimental situation is the only correct way to test the significance of an observed value.
\end{abstract}

\section{INTRODUCTION}

IN this paper, we consider the bias, due to sample size, of some estimators of the relative viabilities of different types in a population. The existence of this kind of bias has long ago been noted. Its approximate value has been given by Haldane (1956) in a particular case, and by Manly (1972) in the context of the mark-release-recapture method. But, as far as we know, there has been no systematic study of its importance and characteristics. The methods of estimation in general use do not take it into account, probably because the investigators believed that they were dealing with a "large sample". Insufficient attention has been given to the question: what is a "large sample"? There is no general answer; the sample size to be considered as "large" depends on the algebraic form of the estimator and on the frequencies of the types in the population.

In many studies, particularly of enzyme polymorphism the size of the sample is very rarely more than 100 , and we shall show that such a sample size does not allow one to neglect the bias. Several methods of estimating viability, as part of the total fitness are in current use in studies of Drosophila (Spiess, 1958, 1979; Anxolabéhère, 1976).

The experimental approach is the following. Two random samples $A$ and B of eggs are taken in a population and grown with or without competition respectively. For an autosomal diallelic locus, the number of adults of each of the three possible genotypes $A_{1} A_{1}, A_{1} A_{2}, A_{2} A_{2}$ is obtained in each condition. The symbols are as follows:

Adults obtained

From sample A: (without competition)

From sample B: (with competition)

\section{Genotypes}

$$
A_{1} A_{1} \quad A_{1} A_{2} \quad A_{2} A_{2} \quad \text { Total }
$$

$\begin{array}{llll}n_{11} & n_{12} & n_{13} & N_{1} \\ n_{21} & n_{22} & n_{23} & N_{2}\end{array}$

* Equipe de recherche associée au C.N.R.S. No. 406: "Etude des mécanismes responsables du maintien du polymorphisme". 
Let $P_{1}, P_{2}, P_{3}$, be the proportions of the three genotypes $A_{1} A_{1}, A_{1} A_{2}$, $A_{2} A_{2}$ in the egg population, $v_{1}, v_{2}, v_{3}$ their survival probabilities without competition up to the adult stage, $v_{1}^{\prime}, v_{2}^{\prime}, v_{3}^{\prime}$ the same probabilities with competition. The adult probabilities of the three genotypes are then:

$\begin{array}{lccc} & \boldsymbol{A}_{1} \boldsymbol{A}_{1} & \boldsymbol{A}_{1} \boldsymbol{A}_{2} & \boldsymbol{A}_{2} \boldsymbol{A}_{2} \\ \text { without competition } & \boldsymbol{P}_{1} v_{1} / \bar{v} & \boldsymbol{P}_{2} v_{2} / \bar{v} & \boldsymbol{P}_{3} v_{3} / \bar{v} \\ \text { with competition } & \boldsymbol{P}_{1} v_{1}^{\prime} / \bar{v}^{\prime} & \boldsymbol{P}_{2} v_{2}^{\prime} / \bar{v}^{\prime} & \boldsymbol{P}_{3} v_{3}^{\prime} / \bar{v}^{\prime}\end{array}$

with

$$
\bar{v}=P_{1} v_{1}+P_{2} v_{2}+P_{3} v_{3} \quad \bar{v}^{\prime}=P_{1} \bar{v}_{1}^{\prime}+P_{2} \bar{v}_{2}^{\prime}+P_{3} \bar{v}_{3}^{\prime}
$$

Comparing the two conditions, we can look at the presence of differential selection by testing the homogeneity of proportions. When differential selection is present, several quantitative measures of it can be used.

In the first method (Bundgaard and Christiansen, 1972; Anxolabéhère, 1976; Johnson, 1976), the estimators of the differential selection

$$
\begin{aligned}
& W_{1}=\frac{n_{21}}{n_{22}} \cdot \frac{n_{12}}{n_{11}} \\
& W_{3}=\frac{n_{23}}{n_{22}} \cdot \frac{n_{12}}{n_{13}}
\end{aligned}
$$

are the ratios of estimators of the relative viabilities of the homozygotes against the heterozygotes. For instance, $n_{21} / n_{22}$ estimates the relative viability of the $A_{1} A_{1}$ genotype against the $A_{1} A_{2}$ genotype. Then, $W_{1}$ and $W_{3}$ estimate respectively $\sigma_{1}=v_{1}^{\prime} / v_{2}^{\prime} \cdot v_{2} / v_{1}$ and $\sigma_{3}=v_{3}^{\prime} / v_{2}^{\prime} \cdot v_{2} / v_{3}$, the differential selection indices of the homozygous genotypes. It is important to note that these estimators depend as much on relative viabilities without competition as on relative viabilities with competition. Particular attention must then be given to their biological significance.

It is often implicitly considered that there is no selection in the condition without competition, and the estimators of differential selection are then taken as measures of the selection in the condition with competition: $W_{1}$ and $W_{3}$ are so used to estimate $v_{1}^{\prime} / v_{2}^{\prime}$ and $v_{3}^{\prime} / v_{2}^{\prime}$. If for instance, selection on viabilities exists in the A sample, but not in the B sample, the estimations of $W_{1}$ and $W_{3}$ could give the erroneous impression of selection in $\mathbf{B}$.

It is not unrealistic to think of a situation where selection would exist at a given locus in an uncrowded condition, this selection disappearing with high density. Then, the $W_{1}$ and $W_{3}$ estimators, used in this context, could lead erroneously to the conclusion of a selection in the B sample.

In the second method (Polivanov, 1964; Kojima and Tobari, 1969; Yarbrough and Kojima, 1967; Johnson, 1976; Spiess, 1979), Hardy-Weinberg proportions in the sample without competition are assumed. The ratio of the number of homozygotes to heterozygotes in the sample with competition is then divided by the ratio of the panmictic expectation of number 
of homozygotes to heterozygotes in the sample without competition. The allelic frequencies are estimated from this sample. The estimators are:

$$
\begin{aligned}
A_{1} A_{1} \quad X_{1} & =\frac{n_{21}}{n_{22}} \cdot \frac{2\left(2 n_{11}+n_{12}\right)\left(n_{12}+2 n_{13}\right)}{\left(2 n_{11}+n_{12}\right)^{2}} \\
& =2 \cdot \frac{n_{21}}{n_{22}} \cdot \frac{\left(n_{12}+2 n_{13}\right)}{\left(2 n_{11}+n_{12}\right)} \\
A_{2} A_{2} \quad X_{2} & =\frac{n_{23}}{n_{22}} \cdot \frac{2\left(2 n_{11}+n_{12}\right)\left(n_{12}+2 n_{13}\right)}{\left(2 n_{13}+n_{12}\right)^{2}} \\
& =2 \cdot \frac{n_{23}}{n_{22}} \cdot \frac{\left(2 n_{11}+n_{12}\right)}{\left(2 n_{13}+n_{12}\right)}
\end{aligned}
$$

noting that $\left(2 n_{11}+n_{12}\right) / 2 N_{1}$ and $\left(n_{12}+2 n_{13}\right) / 2 N_{2}$ are the estimators of the allelic frequencies $p$ and $q$ in the sample without competition. $X_{1}$ and $X_{3}$ are, in fact, the maximum likelihood estimators of the $v_{1}^{\prime} / v_{2}^{\prime}$ and $v_{3}^{\prime} / v_{2}^{\prime}$ ratios when random mating is assumed, and as such reliable for large samples only.

In a previous paper (Goux and Anxolabéhère, 1980), we showed that indices of sexual isolation and selection in current use are so strongly biased in small samples, and their sampling distribution present such peculiarities, as to need caution in their practical use by investigators. Since that time, our attention has been drawn to the formal similarities in these parameters and the differential selection indices; we consider here the properties of small samples.

\section{BiAsES OF THE CURRENT ESTIMATORS OF DIFFERENTIAL SELECTION}

By using the Taylor's series expanded to the second order at the point $E(a), E(b), E(c), E(d)$, it is easy to show (Goux and Anxolabéhère, 1980) that:

$$
\begin{aligned}
E\left(\frac{a d}{b c}\right)= & \frac{E(a) \cdot E(d)}{E(b) \cdot E(c)}\left[1+\frac{\operatorname{var} b}{E^{2}(b)}+\frac{\operatorname{var} c}{E^{2}(c)}-\frac{\operatorname{cov}(a, b)}{E(a) \cdot E(b)}-\frac{\operatorname{cov}(a, c)}{E(a) \cdot E(c)}\right. \\
& \left.+\frac{\operatorname{cov}(a, d)}{E(a) \cdot E(d)}+\frac{\operatorname{cov}(b, c)}{E(b) \cdot E(c)}-\frac{\operatorname{cov}(b, d)}{E(b) \cdot E(d)}-\frac{\operatorname{cov}(c, d)}{E(c) \cdot E(d)}\right]+\varepsilon
\end{aligned}
$$

This formula will be used to establish the small sample properties of the estimators.

(i) The estimators $W_{1}$ and $W_{3}$

(a) Expectation of $W_{1}$ and $W_{3}$ under the null hypothesis of no selection

If $P_{1}, P_{2}, P_{3}$ are the theoretical frequencies of the three genotypes in the egg population, we have for the adults, under the null hypothesis of 
no selection,

$$
\begin{gathered}
E\left(n_{11}\right)=N_{1} P_{1}, \quad E\left(n_{21}\right)=N_{2} P_{1}, \quad E\left(n_{12}\right)=N_{1} P_{2}, \\
E\left(n_{22}\right)=N_{2} P_{2}, \quad \operatorname{var}\left(n_{11}\right)=N_{1} P_{1}\left(1-P_{1}\right), \quad \operatorname{var}\left(n_{21}\right)=N_{2} P_{1}\left(1-P_{1}\right), \\
\operatorname{var}\left(n_{12}\right)=N_{1} P_{2}\left(1-P_{2}\right), \quad \operatorname{var}\left(n_{22}\right)=N_{2} P_{2}\left(1-P_{2}\right), \\
\operatorname{cov}\left(n_{11}, n_{12}\right)=-N_{1} P_{1} P_{2}, \quad \operatorname{cov}\left(n_{21}, n_{22}\right)=-N_{2} P_{1} P_{2}, \\
\operatorname{cov}\left(n_{11}, n_{21}\right)=\operatorname{cov}\left(n_{11}, n_{22}\right)=\operatorname{cov}\left(n_{12}, n_{21}\right)=\operatorname{cov}\left(n_{12}, n_{22}\right)=0 .
\end{gathered}
$$

From these values, we obtain directly:

$$
E\left(W_{1}\right)=E\left(\frac{n_{21}}{n_{22}} \cdot \frac{n_{12}}{n_{11}}\right)=1+\frac{1}{N_{1} P_{1}}+\frac{1}{N_{2} P_{2}}+\varepsilon
$$

and by substitution

$$
E\left(W_{3}\right)=E\left(\frac{n_{23}}{n_{22}}, \frac{n_{12}}{n_{13}}\right)=1+\frac{1}{N_{1} P_{3}}+\frac{1}{N_{2} P_{2}}+\varepsilon .
$$

The estimators $W_{1}$ and $W_{3}$ are biased and their bias depends on the size of the samples and the genetic composition of the population. They are then frequency-dependent.

The investigators, basing their assumptions on the properties of large samples, compare the observed values of the estimators to unity, 1 being taken as their expectation under the null hypothesis of no differential selection. This is incorrect if the samples are not very large. In the particular case where $N_{1}=N_{2}=N$, as is often the case in electrophoretic experiments, and $P_{1}=p^{2}, P_{2}=2 p q, P_{3}=q^{2}$, (random mating with gene frequencies $p$ and $q$ in eggs).

$$
E\left(W_{1}\right)=1+\frac{1}{N}\left(\frac{1}{p^{2}}+\frac{1}{2 p q}\right)+\varepsilon
$$

with an analogous formula for $E\left(W_{3}\right)$.

Fig. 1 shows the variation of these expectations with sample size and allelic frequency. A simulation of the distribution of $W_{1}$ under the null hypothesis of no selection either in A sample or B sample, shows a strong departure of $\vec{W}_{1}$ from one and the skewness of the distribution (fig. 2).

(b) Expectation of $W_{1}$ and $W_{3}$, with selection.

If $v_{1}^{\prime}, v_{2}^{\prime}, v_{3}^{\prime}$, are the viabilities of the three genotypes in the condition with competition, the expectation of adults numbers are:

$$
E\left(n_{21}\right)=\frac{N_{2} P_{1} v_{1}^{\prime}}{\bar{v}^{\prime}}, \quad E\left(n_{22}\right)=\frac{N_{2} P_{2} v_{2}^{\prime}}{\bar{v}^{\prime}}, \quad E\left(n_{23}\right)=\frac{N_{2} P_{3} v_{3}^{\prime}}{\bar{v}^{\prime}}
$$

and their variances:

$$
\begin{array}{ll}
\operatorname{var}\left(n_{21}\right)=\frac{N_{2} P_{1} v_{1}^{\prime}}{\bar{v}^{\prime}}\left(1-\frac{P_{1} v_{1}^{\prime}}{\bar{v}^{\prime}}\right) & \operatorname{var}\left(n_{22}\right)=\frac{N_{2} P_{2} v_{2}^{\prime}}{\bar{v}^{\prime}}\left(1-\frac{P_{2} v_{2}^{\prime}}{\bar{v}^{\prime}}\right) \\
\operatorname{var}\left(n_{23}\right)=\frac{N_{2} P_{3} v_{3}^{\prime}}{\bar{v}^{\prime}}\left(1-\frac{P_{3} v_{3}^{\prime}}{\bar{v}^{\prime}}\right) & \operatorname{cov}\left(n_{21}, n_{22}\right)=-\frac{N_{2} P_{1} P_{2} v_{1}^{\prime} v_{2}^{\prime}}{\bar{v}^{\prime 2}} .
\end{array}
$$




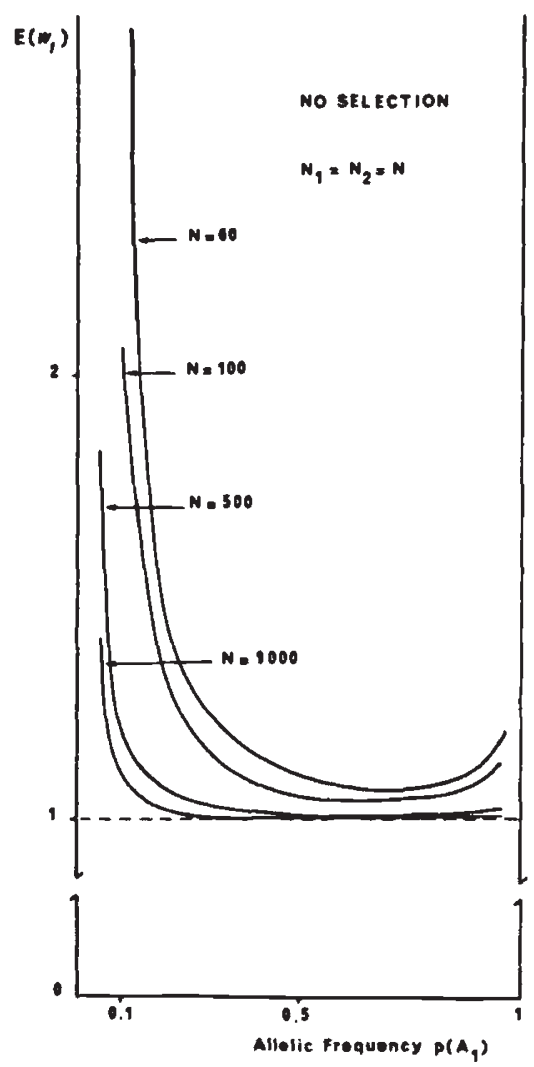

FIG. 1.-Expectation of the differential selection index $W_{1}$ under the null hypothesis of no selection, for different sample sizes $\boldsymbol{N}$ and different values of the allelic frequency (with Hardy-Weinberg proportions among the zygotes).

At the second order of Taylor's series:

$$
\begin{aligned}
& E\left(W_{1}\right)=\frac{v_{1}^{\prime} v_{2}}{v_{2}^{\prime} v_{1}}\left[1+\frac{\bar{v}}{N_{1} P_{1} v_{1}}+\frac{\bar{v}^{\prime}}{N_{2} P_{2} v_{2}^{\prime}}\right]+\varepsilon \\
& E\left(W_{1}\right)=\sigma_{1}\left[1+\frac{\bar{v}}{N_{1} P_{1} v_{1}}+\frac{\bar{v}}{N_{2} P_{2} v_{2}^{\prime}}\right]+\varepsilon .
\end{aligned}
$$

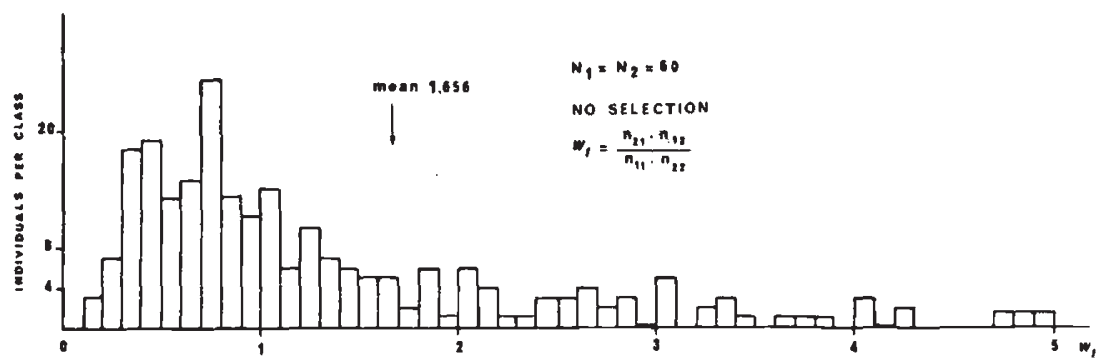

FIG. 2.-Distribution of the differential selection index from 250 drawings, simulated under the null hypothesis of no selection $\left(\sigma_{1}=\sigma_{2}=\sigma_{3}=1\right)$ at the allelic frequency $p\left(A_{1}\right)=0 \cdot 2$, with zygotes in Hardy-Weinberg proportions and $N_{1}=N_{2}=N=60$. 
The similarity with formula (3) is evident. An analogous formula for $E\left(W_{3}\right)$ is obtained directly, by replacing $P_{1}$ by $P_{3}, \sigma_{1}$ by $\sigma_{3}$ and $v_{1}$ by $v_{3}$. It can be shown that $E\left(W_{1}\right)$ and $E\left(W_{3}\right)$ are correlated, the bias of $E\left(W_{1}\right)$ depending on $\sigma_{3}$ and conversely the bias of $E\left(W_{3}\right)$ depending on $\sigma_{1}$.

In the particular case of no selection in the condition without competition, we have:

$$
\sigma_{1}=\frac{v_{1}^{\prime}}{v_{2}^{\prime}}, \quad \sigma_{2}=1, \quad \sigma_{3}=\frac{v_{3}^{\prime}}{v_{2}^{\prime}},
$$

and the formula (4) becomes:

$$
E\left(W_{1}\right)=\sigma_{1}\left[1+\frac{1}{N}\left(\frac{1}{P_{1}}+\frac{\bar{\sigma}}{P_{2}}\right)\right]+\varepsilon
$$

if $N_{1}=N_{2}=N$.

The graph of $E\left(W_{1}\right)$ on allelic frequency in the Hardy-Weinberg conditions is given in fig. 3 .

We have tabulated a particular case, where the true values of the viabilities are respectively linearly frequency-dependent for $A_{1} A_{1}$ and constant for $A_{2} A_{2}$ (fig. 4). The values of $E\left(W_{1}\right)$ in this case are not very

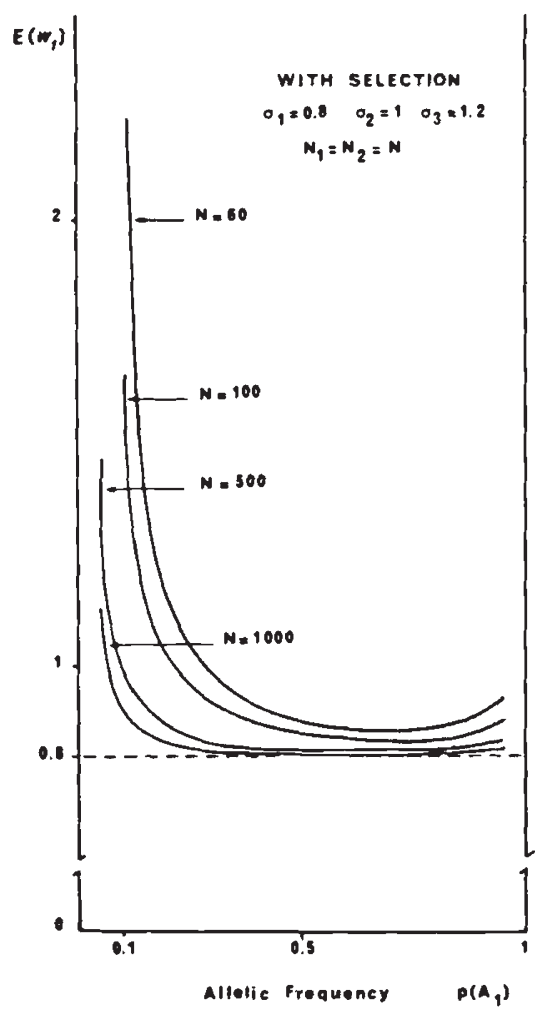

FIG. 3.-Expectations of the differential selection index $W_{1}$ under a particular hypothesis of selection (with constant coefficients, $\sigma_{1}=0 \cdot 8, \sigma_{2}=1, \sigma_{3}=1 \cdot 2$ ) for different sample sizes $N$ and different values of the allelic frequency (with Hardy-Weinberg proportions among the zygotes). 


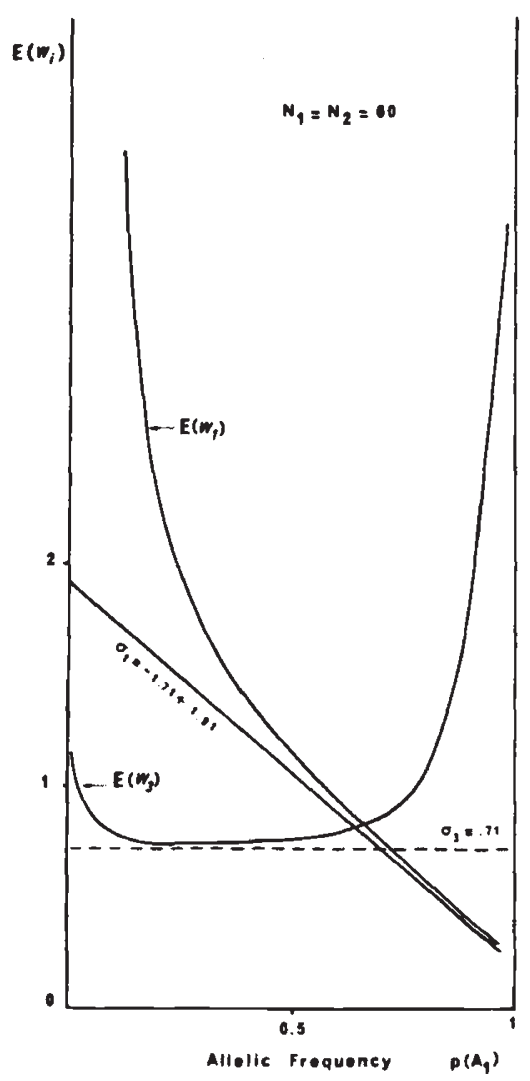

FIG. 4.-Expectations of the differential selection indices $W_{1}$ and $W_{3}$ with a sample size $N_{1}=N_{2}=60$, under a particular frequency-dependent differential selection hypothesis $\left(\sigma_{1}=-1.71 p+1.91, \sigma_{2}=1, \sigma_{3}=0.71\right)$ for different values of the allelic frequency (with Hardy-Weinberg proportions among the zygotes), in the absence of selection where there is no competition.

different, for small $p$, from the values obtained either under the null hypothesis of no selection (fig. 1) or the constant viability selection case (fig. 3). Such a situation can then be misleading.

(ii) The estimators $X_{1}$ and $X_{3}$

If there is no selection either in the $\mathrm{A}$ or in the $\mathrm{B}$ sample,

$$
E\left(X_{1}\right)=2 \frac{P_{1}}{P_{2}} \frac{P_{2}+2 P_{3}}{2 P_{1}+P_{2}}\left[1+\frac{1}{N_{2} P_{2}}+\frac{2}{N_{1}} \frac{4 P_{1} P_{3}+P_{2}\left(1-P_{2}\right)}{\left(P_{2}+2 P_{3}\right)\left(2 P_{1}+P_{2}\right)^{2}}\right]+\varepsilon
$$

and as the $X_{i}$ estimators are constructed under the Hardy-Weinberg hypothesis, we can write by substitution of $P_{1}, P_{2}, P_{3}$ by $p^{2}, 2 p q, q^{2}$, and with $N_{1}=N_{2}=N$

$$
E\left(X_{1}\right)=1+\frac{1}{N} \frac{1+q}{2 p q}+\varepsilon
$$

this expectation being one for $N=\infty$ (fig. 5). 

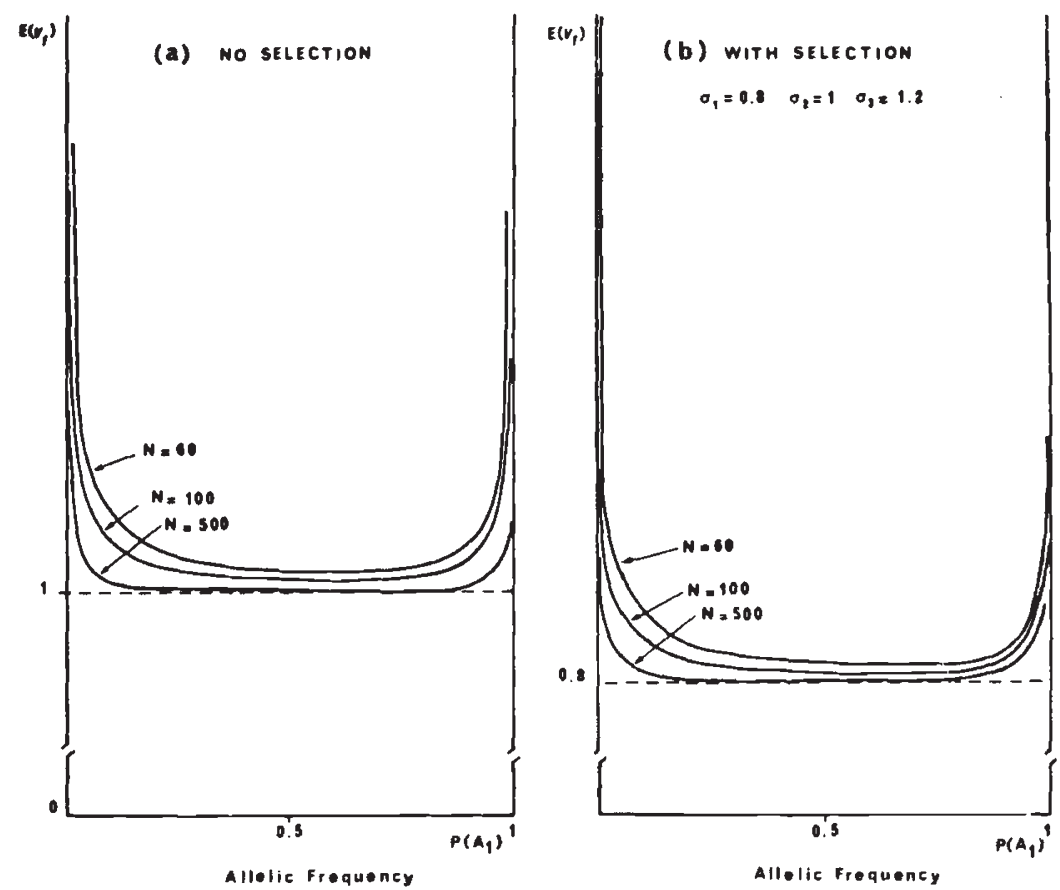

FIG. 5.-Expectations of the differential selection index $X_{1}$ for different sample sizes $N$ and different values of the allelic frequency (with Hardy-Weinberg proportions among the zygotes) (a) under the null hypothesis of no selection, (b) under a particular hypothesis of a differential selection with constant coefficients $\left(\sigma_{1}=0.8, \sigma_{2}=1, \sigma_{3}=1 \cdot 2\right)$, in the absence of selection where there is no competition.

If there is selection, we have, with $\sigma_{1}=v_{1}^{\prime} / v_{2}^{\prime}$,

$$
E\left(X_{1}\right)=2 \sigma_{1} \frac{P_{1}}{P_{2}} \frac{P_{2}+2 P_{3}}{2 P_{1}+P_{2}}\left[1+\frac{\bar{v}^{\prime}}{N_{2} P_{2} v_{2}^{\prime}}+\frac{2\left[4 P_{1} P_{3}+P_{2}\left(1-P_{2}\right)\right]}{\left(P_{2}+2 P_{3}\right)\left(2 P_{1}+P_{2}\right)^{2}}\right]+\varepsilon
$$

admitting no selection in the condition without competition. In HardyWeinberg terms,

$$
E\left(X_{1}\right)=\sigma_{1}\left[1+\frac{1}{N}\left(\frac{\bar{v}^{\prime}}{2 p q v_{2}^{\prime}}+\frac{1}{2 p}\right)\right]+\varepsilon
$$

with $\sigma_{1}=v_{1}^{\prime} / v_{2}^{\prime}$ and $N_{1}=N_{2}=N$.

If $N \rightarrow \infty, E\left(X_{1}\right) \rightarrow \sigma_{1}$.

The same formulae hold for $E\left(X_{3}\right)$ with $P_{1}$ replaced by $P_{3}$ and $\sigma_{1}$ by $\sigma_{3}$. As for the $W_{i}$, we find a frequency-dependence of the expectations for finite $N$, and a strong bias depending on $N$, for small values of allelic frequencies (fig. 5).

It is easy to show that the $X_{i}$ are less biassed than the $W_{i}$, the ratio of the biasses of $E\left(W_{1}\right)$ and $E\left(X_{1}\right)$ being $2 / p$. Nevertheless, the $W_{i}$ are not submitted to the limitation of the Hardy-Weinberg conditions. 


\section{A NEW SET OF ESTIMATORS}

Following Haldane (1956) who suggests adding one to the denominator in estimating viabilities from ratios to give better approximation, we can consider the estimator:

$$
H_{1}=\frac{n_{21} \cdot n_{12}}{\left(n_{22}+1\right)\left(n_{11}+1\right)} .
$$

If we look at each sample as a trinomial drawing, the theoretical proportions of the three genotypes in eggs being $P_{1}, P_{2}, P_{3}$ respectively and the two samples being independently drawn, the probability of a particular experimental result, under the null hypothesis of no selection in both conditions, is:

$$
\begin{aligned}
& P_{0}=P\left(n_{11}, n_{12}, n_{13} / N_{1}\right) \times P\left(n_{21}, n_{22}, n_{23} / N_{2}\right) \\
& =\frac{\left(N_{1}\right) !\left(N_{2}\right) !}{n_{11} ! n_{12} ! n_{13} ! n_{21} ! n_{22} ! n_{23} !} P_{1}^{\left(n_{11}+n_{21}\right)} P_{2}^{\left(n_{12}+n_{22}\right)} P_{3}^{\left(n_{13}+n_{23}\right)}
\end{aligned}
$$

If selection is present, the probability becomes:

$$
\begin{aligned}
P_{s} & =P\left(n_{11}, \ldots, n_{23} / N_{1}, N_{2}\right) \\
& =P_{0} \cdot\left(v_{1}\right)^{n_{11}}\left(v_{2}\right)^{n_{12}}\left(v_{3}\right)^{n_{13}}(\bar{v})^{-N_{1}} \cdot\left(v_{1}^{\prime}\right)^{n_{21}}\left(v_{2}^{\prime}\right)^{n_{22}}\left(v_{3}^{\prime}\right)^{n_{23}}(\bar{v})^{-N_{2}} .
\end{aligned}
$$

If there is no selection in the condition without competition, this formula reduces to:

$$
P_{s}=P_{0} \cdot \sigma_{1}^{n_{21}} \cdot \sigma_{3}^{n_{23}} \cdot(\bar{\sigma})^{-N_{2}}
$$

with

$$
\sigma_{1}=\frac{v_{1}^{\prime}}{v_{2}^{\prime}}, \quad \sigma_{2}=1, \quad \sigma_{3}=\frac{v_{3}^{\prime}}{v_{2}^{\prime}} .
$$

We can then consider the bias in $H_{1}$.

(1) Under the null hypothesis of no selection, the expectation of $n_{21} /\left(n_{22}+1\right)$ is the sum of all possible products

$$
P\left(n_{21}, n_{22}, n_{23} / N_{2}\right) \times \frac{n_{21}}{n_{22}+1}=\frac{N_{2} !}{\left(n_{21}-1\right) !\left(n_{22}+1\right) ! n_{23} !} P_{1}^{n_{21}} P_{2}^{n_{22}} P_{3}^{n_{23}}
$$

and

$$
E\left(\frac{n_{21}}{n_{22}+1}\right)=\frac{P_{1}}{P_{2}} \sum \frac{N_{2} !}{\left(n_{21}-1\right) !\left(n_{22}-1\right) !\left(n_{23}\right) !} P_{1}^{n_{21}-1} P_{2}^{n_{22}+1} P_{3}^{n_{23}}
$$

the sum containing all the terms of $\left(P_{1}+P_{2}+P_{3}\right)^{N_{2}}$, except the terms with $n_{22}=0$.

Then, we have:

$$
E\left(\frac{n_{21}}{n_{22}+1}\right)=\frac{P_{1}}{P_{2}}\left[1-\left(P_{1}+P_{3}\right)^{N_{2}}\right]
$$

In the same way,

$$
E\left(\frac{n_{12}}{n_{11}+1}\right)=\frac{P_{2}}{P_{1}}\left[1-\left(P_{2}+P_{3}\right)^{N_{1}}\right] .
$$


Finally,

$$
E\left(H_{1}\right)=E\left(\frac{n_{21} \cdot n_{12}}{\left(n_{22}+1\right)\left(n_{11}+1\right)}\right)=\left[1-\left(P_{1}+P_{3}\right)^{N_{2}}\right]\left[1-\left(P_{2}+P_{3}\right)^{N_{1}}\right]
$$

(2) When selection is present, the expectations become

with

$$
\begin{aligned}
& E\left(\frac{n_{21}}{n_{22}+1}\right)=\frac{P_{1} v_{1}^{\prime}}{P_{2} v_{2}^{\prime}}\left[1-\left(\frac{P_{1} v_{1}^{\prime}+P_{3} v_{3}^{\prime}}{\bar{v}^{\prime}}\right)^{N_{2}}\right] \\
& E\left(\frac{n_{12}}{n_{11}+1}\right)=\frac{P_{2} v_{2}}{P_{1} v_{1}}\left[1-\left(\frac{P_{2} v_{2}+P_{3} v_{3}}{\bar{v}}\right)^{N_{1}}\right]
\end{aligned}
$$

$$
\begin{gathered}
\sigma_{1}=\frac{v_{1}^{\prime}}{v_{2}^{\prime}} \cdot \frac{v_{2}}{v_{1}} \\
E\left(H_{1}\right)=E\left(\frac{n_{21} \cdot n_{12}}{\left(n_{22}+1\right)\left(n_{11}+1\right)}\right) \\
=\sigma_{1}\left[1-\left(\frac{P_{1} v_{1}^{\prime}+P_{3} v_{3}^{\prime}}{\bar{v}^{\prime}}\right)^{N_{2}}\right]\left[1-\left(\frac{P_{2} v_{2}+P_{3} v_{3}}{\bar{v}}\right)^{N_{1}}\right] .
\end{gathered}
$$

Analogous formulae can be obtained for

$$
H_{3}=\frac{n_{23} \cdot n_{12}}{\left(n_{22}+1\right)\left(n_{13}+1\right)} .
$$

(3) Either without or with selection, it is clear that $E\left(H_{1}\right)$ tends very quickly to 1 or $\sigma_{1}$, when $N_{1}$ and $N_{2}$ increase. Using Haldane's terms, we can then say that the $H_{1}$ estimator is almost unbiased. In the same way, $E\left(H_{3}\right)$ tends to 1 or $\sigma_{3}$.

\section{VARIANCES OF THE $H_{1}$ ESTIMATORS}

The use of the classical formulae on variances of ratios and products shows that, under the null hypothesis of no selection,

$$
\begin{aligned}
\operatorname{var}\left(H_{1}\right)= & \operatorname{var} \frac{n_{21} \cdot n_{12}}{\left(n_{22}+1\right)\left(n_{11}+1\right)} \\
= & \frac{P_{1}^{2}}{P_{2}^{2}}\left[1-\left(P_{1}+P_{3}\right)^{N_{2}}\right]^{2}\left[\frac{N_{1} P_{2}}{N_{1} P_{1}+1}\right]^{2} \cdot B \\
& +\frac{P_{2}^{2}}{P_{1}^{2}}\left[1-\left(P_{2}+P_{3}\right)^{N_{1}}\right]^{2}\left[\frac{N_{2} P_{1}}{N_{2} P_{2}+1}\right]^{2} \cdot A \\
& +\left[\frac{N_{2} P_{1}}{N_{2} P_{2}+1}\right]^{2}\left[\frac{N_{1} P_{2}}{N_{1} P_{1}+1}\right]^{2} \cdot A \cdot B
\end{aligned}
$$

with

$$
\begin{aligned}
& A=\frac{N_{2} P_{1}\left(1-P_{1}\right)}{\left(N_{2} P_{1}\right)^{2}}+\frac{N_{2} P_{2}\left(1-P_{2}\right)}{\left(N_{2} P_{2}+1\right)^{2}}+\frac{2 N_{2} P_{1} P_{2}}{N_{2} P_{1}\left(N_{2} P_{2}+1\right)} \\
& B=\frac{N_{1} P_{2}\left(1-P_{2}\right)}{\left(N_{1} P_{2}\right)^{2}}+\frac{N_{1} P_{1}\left(1-P_{1}\right)}{\left(N_{1} P_{1}+1\right)^{2}}+\frac{2 N_{1} P_{1} P_{2}}{N_{1} P_{2}\left(N_{1} P_{1}+1\right)}
\end{aligned}
$$

annroximatelv. 
But owing to the intrinsic asymmetry of the distribution of $H_{1}$, the use of classical symmetric confidence intervals, restricted to situations where normality can be accepted, is possible only with large samples. If it is not the case, the true distribution of $H_{i}$ must be obtained by tabulation of (8).

In the case where $\left(P_{1}+P_{3}\right)^{N_{2}} \sim 0,\left(P_{2}+P_{3}\right)^{N_{1}} \sim 0$, and if it is possible to admit that $N_{2} P_{2}+1 \sim N_{2} P_{2}$ and $N_{1} P_{1}+1 \sim N_{1} P_{1}$, we can write, with $N_{1}=$ $N_{2}=N$ :

$$
\operatorname{var}\left(H_{1}\right)=\frac{2}{N}\left[\frac{1}{P_{1}}+\frac{1}{P_{2}}\right]+\frac{1}{N^{2}}\left[\frac{1}{P_{1}}+\frac{1}{P_{2}}\right]^{2} .
$$

A numerical example, corresponding to a common situation in electrophoretic investigations, is $N=60, P_{1}=0.25, P_{2}=0.50$, which gives $E\left(H_{1}\right) \sim 1$ and $\sqrt{\operatorname{var}\left(H_{1}\right)} \sim 0.447$ under the null hypothesis.

\section{CONCLusion}

In selection experiments, the estimators of relative viabilities in common use are biased when the sample sizes are not very large, because they are based on ratios of numbers. Their bias depends on sample size and genotypic frequencies, and then is frequency dependent. We present new almost unbiased $H_{i}$ estimators, free of the Hardy-Weinberg condition, deducing from their distribution approximate formulae for their variances. Nevertheless, the intrinsic asymmetry of their distribution often prevents the use of classical symmetric confidence intervals and the true distribution must be tabulated to obtain correct intervals.

Our study does not review all methods of estimating viabilities in current use. In particular, investigators (Anxolabéhère, 1971; Kojima and Huang, 1972; Morgan, 1976; Birley and Beardmore, 1977; Snyder and Ayala, 1979) look at the viabilities of two different types by putting known numbers $R_{1}$ and $N_{1}-R_{1}$ of each of two types, on the medium where competition will proceed. The relative viability is then measured by the ratio $A^{\prime} / B^{\prime}$ of the survivors of each of the two types, after selection. The underlying probability distribution is hypergeometric and it is easy to show that, under the null hypothesis of no selection:

$$
E\left(\frac{A^{\prime}}{B^{\prime}}\right)=\frac{R_{1}}{N_{1}-R_{1}}\left[1+\frac{1}{N}\left(\frac{N_{1}-N}{N_{1}-R_{1}} \frac{N_{1}}{N_{1}-1}\right)\right]+\varepsilon
$$

where $N=A^{\prime}+B^{\prime}$.

The estimator $A^{\prime} / B^{\prime}$ is then biased, the bias depending on $N$ and on the relative frequencies of the input types. The importance of this bias will be considered elsewhere, in the context of a critique of the regression methods used in experiments advocating the existence of a frequencydependent selection.

In the experimental system studied in this paper the samples are not constitutively small, as was the case in our previous work on isolation and sexual selection indices (Goux and Anxolabéhère, 1980). Nevertheless, owing to experimental constraints, the sample size is not very large $(N=60$ is widely used). This is the reason why the maximum likelihood estimators can lead to erroneous conclusions. If not taken into account, the bias can easily lead to the erroneous conclusion of a true frequency-dependent effect on selective values. 
Acknowledgements. - We wish to thank our referee for the suggestion of using Haldane's correction to obtain the almost unbiased estimators $H_{i}$.

\section{REFERENCES}

ANXolabehere, D. 1971. Sélection larvaire et fréquence génique chez Drosophila melanogaster. Heredity, 26, 9-18.

ANXOLABEHERE, D. 1976. Heterosis overdominance and frequency-dependent selection in Drosophila melanogaster at the sepia locus. Evolution, 30, 523-534.

BIRLEY, A. J., AND BEARDMORE. J. A. 1977. Genetical composition, temperature, density and selection in an enzyme polymorphism. Heredity, 39, 133-144.

BUNDGAARD, J., AND CHRISTIANSEN, F. B. 1972. Dynamics of polymorphisms: I. Selection components in an experimental population of Drosophila melanogaster. Genetics, 71, 439-460.

GOUX, J. M., AND ANXOlabehere, D. 1980. The measurement of sexual isolation and selection: a critique. Heredity, 45, 255-262.

HALDANE, J. B. S. 1956. The estimation of viabilities. J. Genet., 54, 294-296.

JOHNSON, C. 1976. Introduction to Natural Selection. University Park Press.

KOJIMA, K. I., AND HUANG, S. L. 1972. Effects of population density on the frequencydependent selection in the Esterase-6 locus of Drosophila melanogaster. Evolution, 26, 313-321.

KOJIMA, K. I., AND TOBARI, Y. N. 1969. Selective models associated with karyotypes in Drosophila ananassae. II. Heterosis and frequency-dependent selection. Genetics, 63, 639-651.

MANLY, B. F. J. 1972. Estimating selective values from field data. Biometrics, 28, 1115-1125.

MORGAN, PH. 1976. Frequency-dependent selection at two enzyme loci in Drosophila melanogaster. Nature, 263, 765-766.

POLIVANOV, s. 1964. Selection in experimental populations of Drosophila melanogaster with different genetic backgrounds. Genetics, 50, 81-100.

SNYDER, T. P., AND AYALA, F. 1979. Frequency-dependent selection at the PGM-1 locus of Drosophila pseudoobscura. Genetics, 92, 995-1003.

SPIESS, E. B. 1958. Chromosomal adaptive polymorphism in Drosophila persimilis. II. Effect of population cage conditions on the life cycle components. Evolution, 12, 234-245.

SPIESS, E. B. 1979. Genes in Populations. John Wiley and Sons.

YARBROUGH, K., AND KOJIMA, K. I. 1967. The mode of selection at the Esterase-6 locus in cage populations of Drosophila melanogaster. Genetics, 57, 677-686. 